\title{
Blow-up rates and uniqueness of entire large solutions to a semilinear elliptic equation with nonlinear convection term
}

\section{Bo Li ${ }^{1 *}$ (D) and Haitao Wan²}

Correspondence: libo_yt@163.com 'School of Mathematics and Information Science, Yantai University, Yantai, P.R. China Full list of author information is available at the end of the article

\begin{abstract}
In this paper, we analyze the blow-up rates and uniqueness of entire large solutions to the equation $\Delta u=a(x) f(u)+\mu b(x)|\nabla u|^{q}, x \in \mathbb{R}^{N}(N \geq 3)$, where $\mu>0, q>0$ and $a, b \in C_{\text {loc }}^{\alpha}\left(\mathbb{R}^{N}\right)(\alpha \in(0,1))$. The weight $a$ is nonnegative, $b$ is able to change sign in $\mathbb{R}^{N}$, and $f \in C^{1}[0, \infty)$ is positive and nondecreasing on $(0, \infty)$ and rapidly or regularly varying at infinity. Additionally, we investigate the uniqueness of entire large solutions.
\end{abstract}

Keywords: Blow-up rates; Entire large solutions; Convection term

\section{Introduction and the main results}

The purpose of this paper is to study the blow-up rates and uniqueness of entire large solutions to the following elliptic equation:

$$
\Delta u=a(x) f(u)+\mu b(x)|\nabla u|^{q}, \quad x \in \mathbb{R}^{N}, u(x)>0,
$$

where $\mu>0, q \in(0,2]$ and $u$ is an entire large solution of Eq. (1.1) when it solves Eq. (1.1) and $u(x) \rightarrow \infty$ as $|x| \rightarrow \infty$. The nonlinearity $f$ is such that

$\left(\mathbf{f}_{1}\right) f \in C^{1}[0, \infty), f(0)=0, f^{\prime}(t) \geq 0$ and $f(t)>0$ for $t>0$ are satisfied; and

$\left(\mathbf{f}_{2}\right)$ the Keller-Osserman condition

$$
\int_{1}^{\infty}(F(t))^{-1 / 2} d t<\infty
$$

holds, and there exists $C_{f} \in[1, \infty)$ such that

$$
\lim _{t \rightarrow \infty}\left((F(t))^{1 / 2}\right)^{\prime} \int_{t}^{\infty}(F(s))^{-1 / 2} d s=C_{f}, \quad F(t)=\int_{0}^{t} f(s) d s
$$

The weight $a$ is such that

$\left(\mathbf{a}_{1}\right) a \in C_{\text {loc }}^{\alpha}\left(\mathbb{R}^{N}\right)(\alpha \in(0,1))$ is positive in $\mathbb{R}^{N}$; and

$\left(\mathbf{a}_{2}\right)$ there exist $k_{1} \in \mathcal{K}$ and positive constant $\lambda_{1} \in[2,2(N-1))$ such that

$$
0<a_{1}:=\liminf _{|x| \rightarrow \infty} \frac{a(x)}{|x|^{-\lambda_{1}} k_{1}(|x|)} \leq a_{2}:=\limsup _{|x| \rightarrow \infty} \frac{a(x)}{|x|^{-\lambda_{1}} k_{1}(|x|)}<\infty
$$

(c) The Author(s) 2018. This article is distributed under the terms of the Creative Commons Attribution 4.0 International License (http://creativecommons.org/licenses/by/4.0/), which permits unrestricted use, distribution, and reproduction in any medium, provided you give appropriate credit to the original author(s) and the source, provide a link to the Creative Commons license, and indicate if changes were made. 
where

$$
\int_{t_{0}}^{\infty} \frac{k_{1}(s)}{s} d s<\infty, \quad \text { if } \lambda_{1}=2
$$

$\mathcal{K}$ denotes the set of Karamata functions $k$ defined on $\left[t_{0}, \infty\right)$ by

$$
k(t):=c \exp \left(\int_{t_{0}}^{t} \frac{y(s)}{s} d s\right), \quad t \geq t_{0}>0
$$

with $c>0$ and $y \in C\left(\left[t_{0}, \infty\right)\right)$ such that $\lim _{t \rightarrow \infty} y(t)=0$.

The weight $b$ is such that

(b) $b \in C_{\text {loc }}^{\alpha}\left(\mathbb{R}^{N}\right)(\alpha \in(0,1))$ may change sign in $\mathbb{R}^{N}$; and

$\left(\mathbf{b}_{2}\right)$ there exist $k_{2} \in \mathcal{K}$ and positive constant $\lambda_{2}$ such that

$$
0<b_{1}:=\liminf _{|x| \rightarrow \infty} \frac{|b(x)|}{|x|^{-\lambda_{2}} k_{2}(|x|)} \leq b_{2}:=\limsup _{|x| \rightarrow \infty} \frac{|b(x)|}{|x|^{-\lambda_{2}} k_{2}(|x|)}<\infty
$$

where $\mathcal{K}$ is defined in $\left(\mathbf{a}_{2}\right)$.

Let $\Omega$ be a bounded domain with a $C^{2}$-boundary in $\mathbb{R}^{N}$ or $\Omega=\mathbb{R}^{N}$. The existence, nonexistence, uniqueness and asymptotic behavior of large solutions to Eq. (1.1) have been investigated extensively by many authors in different contexts. When $\Omega$ is a bounded domain, a "large solution" is understood as a solution $u$ of Eq. (1.1) on $\Omega$ such that $\lim _{x \rightarrow \partial \Omega} u(x)=\infty$.

Now, we review these studies and divide them into the following two sets:

Part $I(\mu=0)$. When $\Omega$ is a bounded domain with a $C^{2}$-boundary, $b \equiv 1$ in $\Omega \subseteq \mathbb{R}^{N}$ $(N=2)$ and $f(u)=e^{u}$; Bieberbach [5] first studied the existence, uniqueness and asymptotic behavior of large solutions to Eq. (1.1). In this case, Eq. (1.1) plays an important role in the theory of Riemannian surfaces of constant negative curvature and in the theory of automorphic functions. Later, Rademacher [40] used the ideas of Bieberbach to prove that the results still hold for $N=3$. When $f(u)=e^{u}$ and $b$ is continuous and strictly positive on $\bar{\Omega}$, Lazer and McKenna [30] extended the above results to a bounded domain $\Omega$ in $\mathbb{R}^{N}$ $(N \geq 1)$ with a uniform outer sphere. However, Keller [26] and Osserman [39] conducted systematic research on Eq. (1.1) and obtained the following important results:

(i) If $\Omega \subseteq \mathbb{R}^{N}$ is a bounded domain, $b \equiv 1$ on $\bar{\Omega}$, and $f$ satisfies (f $\mathbf{f}_{\mathbf{1}}$ ), then Eq. (1.1) has a classical large solution if and only if the Keller-Osserman condition $\left(\mathbf{f}_{2}\right)$ holds.

(ii) If $b \equiv 1$ in $\mathbb{R}^{N}$ and $f$ satisfies $\left(\mathbf{f}_{\mathbf{1}}\right)$, then Eq. (1.1) has an entire large solution if and only if the following condition holds:

$$
\int_{1}^{\infty}(2 F(t))^{-1 / 2} d t<\infty
$$

When $\Omega$ is a bounded domain, $b \equiv 1$ in $\Omega$ and $f(u)=u^{\frac{N+2}{N-2}}$ with $N>2$, Loewner and Nirenberg [32] were motivated by certain geometric problems to prove that Eq. (1.1) has a unique positive classical large solution $u$ satisfying

$$
\lim _{d(x) \rightarrow 0} u(x)(d(x))^{\frac{N-2}{2}}=\left(\frac{N(N-2)}{4}\right)^{\frac{N-2}{4}} .
$$


Later, Bandle and Marcus [4] further studied the exact asymptotic behavior and uniqueness of a large solution to Eq. (1.1) when $f$ is convex on $(0, \infty)$, i.e., $s \mapsto f(s)$ is increasing on $(0, \infty)$. However, when $b$ is a large constant, by obtaining good boundary layer estimates for $b$, Du and Guo [14] proved that the large solution is unique for much weaker assumptions as regards $f$, i.e., $s \mapsto f(s)$ is increasing on $(T, \infty)$ for some large constant $T$. For other insights into the existence, uniqueness, multiplicity and asymptotic behavior of large solutions to Eq. (1.1) with $\mu=0$ in bounded domains, please refer to $[8,15,17,24$, $29,31,33-36,50,52-54]$ and the references therein.

Now, let us return to entire large solutions of Eq. (1.1) with $\mu=0$. Oleinik [38] and Sattinger [44] studied the nonexistence of entire positive solutions of Eq. (1.1). Later, the existence and asymptotic behavior of entire large solutions was first described by $\mathrm{Ni}$ in [37]. For $f(u)=u^{\gamma}$ with $\gamma>1$, Cheng and Ni [7] studied the existence and uniqueness of the maximum positive solution and completely classified all possible positive solutions. Later, Lair [27], Cîrstea and Rădulescu [10], and Tao and Zhang [46] further investigated the existence of entire large solutions of Eq. (1.1). In particular, when $f$ exhibits sublinear growth at infinity, Lair [28] proved that if $b$ satisfies

$$
\int_{0}^{\infty} r\left(\phi_{1}(r)-\phi_{2}(r)\right) \varphi(r) d r<\infty
$$

where

$$
\varphi(r)=\exp \left(\hbar \int_{0}^{r} \tau \phi_{2}(\tau) d \tau\right)
$$

with

$$
\phi_{1}(r):=\max _{|x|=r} b(x), \quad \phi_{2}(r):=\min _{|x|=r} b(x)
$$

then Eq. (1.1) has entire large solutions if and only if

$$
\int_{0}^{\infty} r \phi_{2}(r) d r=\infty
$$

In [16], Dupaigne et al. established the existence of a minimum solution $u$ and noted that if $u$ is an unbounded solution, then it blows up at infinity. In particular, they also showed that the entire large solution of Eq. (1.1) is unique for some appropriate assumptions as regards $f$ and $b$. Inspired by the ideas of the authors in $[1,17,18]$, Wan $[47,48]$ and Wan et al. [49] used a perturbation method and a truncation technique to study the asymptotic behavior and uniqueness of entire large solutions of Eq. (1.1). In particular, the authors in [49] found some necessary and sufficient conditions for these rapidly and regularly varying functions and proved some sharp uniqueness results for entire large solutions when $f$ belongs to a more general class of functions.

Part II $(\mu \neq 0)$. When $f(u)=u^{\gamma}(\gamma>1)$ or $f(u)=e^{u}, \mu=1$ and the weights $a, b \equiv 1$ in $\Omega$, Bandle and Giarrusso [3] studied the existence and asymptotic behavior of large solutions to Eq. (1.1). Later, Giarrusso [21] and [22] further extended the above results when $f$ belongs to a more general class of functions. When $\Omega=\mathbb{R}^{N}, f(u)=u^{\gamma}(\gamma>1)$ and $\mu b= \pm 1$ in $\mathbb{R}^{N}$, Lair investigated the existence of entire large solutions of Eq. (1.1). When $f$ satisfies 
$\left(\mathbf{f}_{\mathbf{1}}\right)$, Ghergu and Rădulescu [19] established the existence and nonexistence of entire large solutions of an one-dimensional logistic problem. When $\Omega$ is a smooth bounded domain or $\Omega=R^{N}$, Ghergu and Rădulescu [20] studied the existence and nonexistence of large solutions of Eq. (1.1) provided the nonlinearity $f$ satisfies $\sup _{s \geq 1} f(s) / s<\infty$ and the weight $\mu b \equiv-1$ on $\Omega$. In particular, they supplied, for the case $\Omega=R^{N}$, a necessary and sufficient condition for the existence of a positive solution that blows up at infinity. In a different direction, Cîrstea and Rădulescu [9, 11-13] first introduced Karamata regular variation theory to study the boundary behavior and uniqueness of large solutions of Eq. (1.1) in a bounded domain. This contributed to knowledge in some new directions and enabled us to obtain a significant amount of information about the qualitative behavior of large solutions in a general framework. Using this approach, Zhang [51] and Huang et al. [25] determined the exact asymptotic behavior of large solutions to Eq. (1.1) with $\mu= \pm 1$ in a bounded domain.

Recently, Rhouma and Drissi [42] studied the existence of positive radial solutions and entire large solutions to a class of $p$-Laplacian equations. Santos et al. [43] studied the existence of entire large solutions to a class of variable-index Laplacian equations. Alves, Santos and Zhou [2] established a new comparison principle to investigate the existence of entire large solutions to Eq. (1.1) that depend on a nonnegative real parameter. Moreover, inspired by the idea of Mitidieri and Pohozaev [34], they established the nonexistence of entire large solutions by constructing a test function, which is null in the exterior of appropriate balls in $\mathbb{R}^{N}$.

Motivated by the work of [2] and [47-49], in this paper, we determine the exact asymptotic behavior of entire large solutions to Eq. (1.1) at infinity in $\mathbb{R}^{N}$, and we show that the convection term $\mu b(x)|\nabla u(x)|^{q}$ does not affect the asymptotic behavior under certain conditions. Moreover, we prove that the entire large solution is unique using its asymptotic behavior.

Our results are summarized as follows.

Theorem 1.1 Let $f$ satisfy $\left(\mathbf{f}_{\mathbf{1}}\right)-\left(\mathbf{f}_{\mathbf{2}}\right)$ with $(q-1) C_{f}<1$, weight a satisfy $\left(\mathbf{a}_{\mathbf{1}}\right)-\left(\mathbf{a}_{2}\right)$, weight $b$ satisfy $\left(\mathbf{b}_{1}\right)-\left(\mathbf{b}_{2}\right)$, and $\lambda_{2}>\frac{\lambda_{1}(2-q)}{2}$. Moreover, we further assume that one of the following conditions holds:

$\left(\mathbf{S}_{1}\right) \quad b \leq 0$ in $\mathbb{R}^{N}$ and $q \in(0,2]$; and

$\left(\mathbf{S}_{2}\right)$ b can change sign in $\mathbb{R}^{N}, q=1$ and $\min \left\{\lambda_{1}, \lambda_{2}\right\} \geq 2$. In particular, if $\lambda_{2}=2$, we need to verify that

$$
\int_{t_{0}}^{\infty} \frac{k_{2}(s)}{s} d s<\infty
$$

Then any entire large solution $u$ of problem (1.1) satisfies

$$
\begin{aligned}
\xi_{2}^{1-C_{f}} & \leq \liminf _{|x| \rightarrow \infty} \frac{u(x)}{\psi\left(\left(\int_{|x|}^{\infty} s^{1-\lambda_{1}} k_{1}(s) d s\right)^{1 / 2}\right)} \\
& \leq \limsup _{|x| \rightarrow \infty} \frac{u(x)}{\psi\left(\left(\int_{|x|}^{\infty} s^{1-\lambda_{1}} k_{1}(s) d s\right)^{1 / 2}\right)} \leq \xi_{1}^{1-C_{f}},
\end{aligned}
$$


where $\psi$ is uniquely determined by

$$
\int_{\psi(t)}^{\infty}(2 F(s))^{-1 / 2} d s=t, \quad t>0
$$

and

$$
\xi_{i}=\left(\frac{4 a_{i} C_{f}}{\left(\lambda_{1}-2\right)\left(C_{f}-1\right)+2(N-2)}\right)^{1 / 2}, \quad i=1,2
$$

In particular, if $C_{f}=1$ in $\left(\mathbf{f}_{2}\right)$, then any entire large solution $u$ of problem (1.1) satisfies

$$
\lim _{|x| \rightarrow \infty} \frac{u(x)}{\psi\left(\left(\int_{|x|}^{\infty} s^{1-\lambda_{1}} k_{1}(s) d s\right)^{1 / 2}\right)}=1 .
$$

Remark 1.1 In Theorem 1.1, $\left(\mathbf{S}_{\mathbf{1}}\right)$ and $\left(\mathbf{S}_{\mathbf{2}}\right)$ are auxiliary conditions that guarantee the existence of entire large solutions to Eq. (1.1) (please refer to [2]).

Remark 1.2 In Theorem 1.1, $C_{f}(q-1)<1$ implies that if $C_{f}=1$, then $q \neq 2$.

Theorem 1.2 Let a satisfy $\left(\mathbf{a}_{\mathbf{1}}\right), b$ satisfy $\left(\mathbf{b}_{\mathbf{1}}\right)$ and the following condition:

(b) $b \geq 0$ in $\mathbb{R}^{N}, q \geq 1$ or $b \leq 0$ in $\mathbb{R}^{N}, 0<q<1$.

Additionally, let $f$ satisfy $\left(\mathbf{f}_{\mathbf{1}}\right)$ and the following condition:

$\left(\mathbf{f}_{3}\right) t \mapsto \frac{f(t)}{t}$ is nondecreasing on $(0, \infty)$.

Assume that $u_{1}$ and $u_{2}$ are arbitrary entire large solutions of Eq. (1.1) that satisfy

$$
\lim _{|x| \rightarrow \infty} \frac{u_{1}(x)}{u_{2}(x)}=1
$$

Then $u_{1} \equiv u_{2}$ in $\mathbb{R}^{N}$.

The outline of this paper is as follows. The proofs of Theorem 1.1 and Theorem 1.2 are given in Sects. 2 and 3. Then we list some bases for Karamata regular variation theory and present some auxiliary results in Appendices 1 and 2.

\section{Proof of Theorem 1.1}

In this section, we prove Theorem 1.1.

Proof Let $\varepsilon \in\left(0, b_{1} / 2\right)$ and

$$
\tau_{1}=\left(\xi_{1}^{2}-\varepsilon \xi_{1}^{2} / b_{1}\right)^{1 / 2}, \quad \tau_{2}=\left(\xi_{2}^{2}+\varepsilon \xi_{2}^{2} / b_{2}\right)^{1 / 2} .
$$

We see that

$$
(1 / 2)^{1 / 2} \xi_{1}<\tau_{1}<\tau_{2}<(3 / 2)^{1 / 2} \xi_{2}
$$

and

$$
\lim _{\varepsilon \rightarrow 0} \tau_{1}=\xi_{1}, \quad \lim _{\varepsilon \rightarrow 0} \tau_{2}=\xi_{2} .
$$


For any constant $R>t_{0}$, we define

$$
\Omega_{R}:=\left\{x \in \mathbb{R}^{N}:|x|>R\right\},
$$

where $t_{0}$ is given by the definition of $\mathcal{K}$ in $\left(\mathbf{a}_{2}\right)$.

From $\left(\mathbf{f}_{1}\right)-\left(\mathbf{f}_{2}\right)$ with $(q-1) C_{f}<1,\left(\mathbf{a}_{1}\right)-\left(\mathbf{a}_{2}\right)$ and $\left(\mathbf{b}_{1}\right)-\left(\mathbf{b}_{2}\right)$ with $\lambda_{2}>\frac{\lambda_{1}(2-q)}{2}$, Propositions 3-4, and Lemma B.2(iii), we see that there exists a sufficiently large $R_{\varepsilon}>0$ and a sufficiently small $\delta_{\varepsilon}>0$ corresponding to $\varepsilon>0$ such that, for $(x, r) \in \Omega_{R_{\varepsilon}} \times\left(0,2 \delta_{\varepsilon}\right)$,

$$
\begin{aligned}
& \frac{|x|^{2-\lambda_{1}} k_{1}(|x|)}{\int_{|x|}^{\infty} s^{1-\lambda_{1}} k_{1}(s) d s}+2\left(N-\lambda_{1}\right)+\frac{2|x| k_{1}^{\prime}(|x|)}{k_{1}(|x|)}>0 \\
& \left|\frac{\tau_{i}^{2}}{4} \frac{|x|^{2-\lambda_{1}} k_{1}(|x|)}{\int_{|x|}^{\infty} s^{1-\lambda_{1}} k_{1}(s) d s}-\frac{\tau_{i}^{2}\left(\lambda_{1}-2\right)}{4}\right|+\mid-\frac{\tau_{i}^{2}}{4}\left(\frac{|x|^{2-\lambda_{1}} k_{1}(|x|)}{\int_{|x|}^{\infty} s^{1-\lambda_{1}} k_{1}(s) d s}+2\left(N-\lambda_{1}\right)\right. \\
& \left.\quad+\frac{2|x| k_{1}^{\prime}(|x|)}{k_{1}(|x|)}\right) \frac{\psi^{\prime}(r)}{r \psi^{\prime \prime}(r)}-\frac{\left(2(N-1)-\lambda_{1}\right) \tau_{i}^{2}}{4 C_{f}} \mid<\varepsilon / 3, \\
& \left|\mu\left(\frac{\tau_{i}}{2}\right)^{q} \frac{b(x)}{|x|^{-\lambda_{2}} k_{2}(|x|)} \frac{\left(-\psi^{\prime}(r)\right)^{q}\left(\int_{|x|}^{\infty} s^{1-\lambda_{1}} k_{1}(s) d s\right)^{\frac{-q}{2}}|x|^{q\left(1-\lambda_{1}\right)+\lambda_{1}-\lambda_{2}} k_{1}^{q}(|x|)}{\psi^{\prime \prime}(r)} \frac{k_{2}(|x|)}{k_{1}(|x|)}\right| \\
& \quad=\mu\left(\frac{\tau_{i}}{2}\right)^{q} \frac{b(x)}{|x|^{-\lambda_{2}} k_{2}(|x|)} \frac{k_{2}(|x|)}{k_{1}(|x|)} \cdot \frac{\left(-\psi^{\prime}(r)\right)}{r \psi^{\prime \prime}(r)} \cdot r\left(-\psi^{\prime}(r)\right)^{q-1} \cdot\left(\int_{|x|}^{\infty} s^{1-\lambda_{1}} k_{1}(s) d s\right)^{\frac{-q}{2}} \\
& \quad \times|x|^{q\left(1-\lambda_{1}\right)+\lambda_{1}-\lambda_{2}} k_{1}^{q}(|x|)<\varepsilon / 3
\end{aligned}
$$

and

$$
|x|^{-\lambda_{1}} k_{1}(|x|)\left(a_{1}-\varepsilon / 3\right)<a(x)<|x|^{-\lambda_{1}} k_{1}(|x|)\left(a_{2}+\varepsilon / 3\right) .
$$

Take

$$
\sigma \in\left(0, \delta_{\varepsilon}\right) \quad \text { with } \sigma<(1 / 2)^{1 / 2} \xi_{1}\left(\int_{R_{\varepsilon}}^{\infty} s^{1-\lambda_{1}} k_{1}(s) d s\right)^{1 / 2}
$$

and let $u$ be an arbitrary entire large solution of problem (1.1).

Define

$$
D_{-}^{\sigma}:=\Omega_{R_{\varepsilon}} \backslash \Omega_{-}^{\sigma}, \quad D_{+}^{\sigma}:=\Omega_{R_{\varepsilon}} \backslash \Omega_{+}^{\sigma},
$$

where

$$
\Omega_{-}^{\sigma}:=\left\{x \in \Omega_{R_{\varepsilon}}: \tau_{1}\left(\int_{|x|}^{\infty} s^{1-\lambda_{1}} k_{1}(s) d s\right)^{1 / 2} \leq \sigma\right\}
$$

and

$$
\Omega_{+}^{\sigma}:=\left\{x \in \Omega_{R_{\varepsilon}+r_{0}}: \psi\left(\tau_{2}\left(\int_{|x|}^{\infty} s^{1-\lambda_{1}} k_{1}(s) d s\right)^{1 / 2}+\sigma\right) \leq u(x)\right\}
$$


where $r_{0}$ is a sufficiently large constant that $\Omega_{+}^{\sigma}$ is an exterior domain of the ball with $R_{\varepsilon}+r_{0}$ as the radius and the origin as the center. By the definitions of $\Omega_{ \pm}^{\sigma}$, we see that $D_{ \pm}^{\sigma}$ are annular domains.

Let

$$
\bar{u}_{\varepsilon}(x)=\psi\left(\tau_{1}\left(\int_{|x|}^{\infty} s^{1-\lambda_{1}} k_{1}(s) d s\right)^{1 / 2}-\sigma\right), \quad x \in D_{-}^{\sigma}
$$

and

$$
\underline{u}_{\varepsilon}=\psi\left(\tau_{2}\left(\int_{|x|}^{\infty} s^{1-\lambda_{1}} k_{1}(s) d s\right)^{1 / 2}+\sigma\right), \quad x \in D_{+}^{\sigma} .
$$

Indeed, we can always adjust the above $\delta_{\varepsilon}>0$ to ensure that

$$
(3 / 2)^{1 / 2} \xi_{2}\left(\int_{|x|}^{\infty} s^{1-\lambda_{1}} k_{1}(s) d s\right)^{1 / 2}<\delta_{\varepsilon}, \quad x \in \Omega_{R_{\varepsilon}}
$$

By a straightforward calculation combined with (2.1)-(2.4), we see that, for $x \in D_{-}^{\sigma}$,

$$
\begin{aligned}
\Delta \bar{u}_{\varepsilon} & -a(x) f\left(\bar{u}_{\varepsilon}\right)-\mu b(x)\left|\nabla \bar{u}_{\varepsilon}\right|^{q} \\
= & \psi^{\prime \prime}\left(\tau_{1}\left(\int_{|x|}^{\infty} s^{1-\lambda_{1}} k_{1}(s) d s\right)^{1 / 2}-\sigma\right)|x|^{-\lambda_{1}} k(|x|)\left[\frac{\tau_{1}^{2}}{4} \frac{|x|^{2-\lambda_{1}} k_{1}(|x|)}{\int_{|x|}^{\infty} s^{1-\lambda_{1}} k_{1}(s) d s}\right. \\
& +\frac{\tau_{1}^{2}}{4}\left(\frac{|x|^{2-\lambda_{1}} k_{1}(|x|)}{\int_{|x|}^{\infty} s^{1-\lambda_{1}} k_{1}(s) d s}+2\left(N-\lambda_{1}\right)+\frac{2|x| k_{1}^{\prime}(|x|)}{k_{1}(|x|)}\right) \\
& \times \frac{-\psi^{\prime}\left(\tau_{1}\left(\int_{|x|}^{\infty} s^{1-\lambda_{1}} k_{1}(s) d s\right)^{1 / 2}-\sigma\right)}{\tau_{1}\left(\int_{|x|}^{\infty} s^{1-\lambda_{1}} k_{1}(s) d s\right)^{1 / 2} \psi^{\prime \prime}\left(\tau_{1}\left(\int_{|x|}^{\infty} s^{1-\lambda_{1}} k_{1}(s) d s\right)^{1 / 2}-\sigma\right)}-\frac{a(x)}{|x|^{-\lambda_{1}} k_{1}(|x|)} \\
& -\frac{\left(-\psi^{\prime}\left(\tau_{1}\left(\int_{|x|}^{\infty} s^{1-\lambda_{1}} k_{1}(s) d s\right)^{1 / 2}-\sigma\right)^{q}\left(\int_{|x|}^{\infty} s^{1-\lambda_{1}} k_{1}(s) d s\right)^{\frac{-q}{2}}|x|^{q\left(1-\lambda_{1}\right)+\lambda_{1}-\lambda_{2}} k_{1}^{q}(|x|)\right.}{\psi^{\prime \prime}\left(\tau_{1}\left(\int_{|x|}^{\infty} s^{1-\lambda_{1}} k_{1}(s) d s\right)^{1 / 2}-\sigma\right)} \\
& \left.\times \frac{k_{2}(|x|)}{k_{1}(|x|)} \mu\left(\frac{\tau_{1}}{2}\right)^{q} \frac{b(x)}{|x|^{-\lambda_{2}} k_{2}(|x|)}\right] \\
\leq & \psi^{\prime \prime}(r)|x|^{-\lambda_{1}} k_{1}(|x|)\left[\frac{\tau_{1}^{2}}{4}\left(\frac{|x|^{2-\lambda_{1}} k_{1}(|x|)}{\int_{|x|}^{\infty} s^{1-\lambda_{1}} k_{1}(s) d s}-\left(\lambda_{1}-2\right)\right)\right. \\
& -\frac{\tau_{1}^{2}}{4}\left(\frac{|x|^{2-\lambda_{1}} k_{1}(|x|)}{\int_{|x|}^{\infty} s^{1-\lambda_{1}} k_{1}(s) d s}+2\left(N-\lambda_{1}\right)+\frac{2|x| k_{1}^{\prime}(|x|)}{k_{1}(|x|)}\right) \frac{\psi^{\prime}(r)}{r \psi^{\prime \prime}(r)}-\frac{\left(2(N-1)-\lambda_{1}\right) \tau_{1}^{2}}{4 C_{f}} \\
& \left.-\left(\frac{a(x)}{|x|^{-\lambda_{1}} k_{1}(|x|)}-a_{1}\right)+\tau_{1}^{2}\left(\frac{2(N-2)+\left(C_{f}-1\right)\left(\lambda_{1}-2\right)}{4 C_{f}}\right)-a_{1}\right] \leq 0,
\end{aligned}
$$

where

$$
r=\tau_{1} \int_{|x|}^{\infty} s^{1-\lambda_{1}} k_{1}(s) d s-\sigma,
$$

i.e., $\bar{u}_{\varepsilon}$ is a supersolution of Eq. (1.1) in $D_{-}^{\sigma}$. 
Similarly, we can show that $\underline{u}_{\varepsilon}$ is a subsolution of Eq. (1.1) in $D_{\sigma}^{+}$. We assert that there exists a large constant $M>0$ independent of $\sigma$ such that

$$
u(x) \leq \bar{u}_{\varepsilon}(x)+M, \quad x \in D_{-}^{\sigma}
$$

and

$$
\underline{u}_{\varepsilon}(x) \leq u(x)+M, \quad x \in \Omega_{R_{\varepsilon}}
$$

In fact, we can choose a positive constant $M$ independent of $\sigma$ such that $x \in\left\{x \in \mathbb{R}^{N}:|x|=\right.$ $\left.R_{\varepsilon}\right\}$

$$
u(x) \leq \bar{u}_{\varepsilon}(x)+M
$$

and

$$
\underline{u}_{\varepsilon}(x) \leq u(x)+M \text {. }
$$

Because

$$
u(x)<\bar{u}_{\varepsilon}=\infty, \quad x \in\left\{x \in \mathbb{R}^{N}: \tau_{1}\left(\int_{|x|}^{\infty} s^{1-\lambda_{1}} k_{1}(s) d s\right)^{1 / 2}=\sigma\right\}
$$

we can always choose a sufficiently small $\rho>0$ such that

$$
\sup _{x \in D_{-}^{\sigma}} u(x) \leq \bar{u}_{\varepsilon}(x), \quad x \in D_{-}^{\sigma} \backslash \tilde{D}_{-}^{\sigma},
$$

where

$$
\tilde{D}_{-}^{\sigma}=\Omega_{R_{\varepsilon}} \backslash \tilde{\Omega}_{-}^{\sigma}
$$

with

$$
\tilde{\Omega}_{-}^{\sigma}=\left\{x \in \Omega_{R_{\varepsilon}}: \tau_{1}\left(\int_{|x|}^{\infty} s^{1-\lambda_{1}} k_{1}(s)\right)^{1 / 2} \leq \sigma(1+\rho)\right\} .
$$

Combining (2.9) with (2.11), we obtain

$$
u(x) \leq \bar{u}_{\varepsilon}(x)+M, \quad x \in \partial\left(\tilde{D}_{-}^{\sigma}\right)
$$

In contrast, we conclude, using (2.10) and the definition of $\Omega_{+}^{\sigma}$ (please refer to (2.6)), that

$$
\underline{u}_{\varepsilon}(x) \leq u(x)+M, \quad x \in \partial\left(D_{+}^{\sigma}\right) \text {. }
$$

We note that $u$ and $\underline{u}_{\varepsilon}$ are both subsolutions in $\tilde{D}_{-}^{\sigma}$ and $D_{+}^{\sigma}$. Therefore, using $\left(\mathbf{f}_{\mathbf{1}}\right)$, we see that $\bar{u}_{\varepsilon}+M$ and $u+M$ are supersolutions in $\tilde{D}_{-}^{\sigma}$ and $D_{+}^{\sigma}$, respectively. It follows by Lemma B.3 that

$$
u(x) \leq \bar{u}_{\varepsilon}(x)+M, \quad x \in \tilde{D}_{-}^{\sigma}
$$


and

$$
\underline{u}_{\varepsilon}(x) \leq u(x)+M, \quad x \in D_{+}^{\sigma} .
$$

Indeed, (2.12) combined with (2.11) implies that (2.7) holds, and (2.13) and (2.6) together imply that (2.8) holds.

Now, using (2.7) and (2.8), we show that, for any $x \in \Omega_{R_{\varepsilon}}$, the following hold:

$$
\begin{aligned}
& \frac{u(x)}{\psi\left(\tau_{1}\left(\int_{|x|}^{\infty} s^{1-\lambda_{1}} k_{1}(s) d s\right)^{1 / 2}\right)} \leq 1+\frac{M}{\psi\left(\tau_{1}\left(\int_{|x|}^{\infty} s^{1-\lambda_{1}} k_{1}(s) d s\right)^{1 / 2}\right)} \\
& \frac{u(x)}{\psi\left(\tau_{2}\left(\int_{|x|}^{\infty} s^{1-\lambda_{1}} k_{1}(s) d s\right)^{1 / 2}\right)} \geq 1-\frac{M}{\psi\left(\tau_{2}\left(\int_{|x|}^{\infty} s^{1-\lambda_{1}} k_{1}(s) d s\right)^{1 / 2}\right)}
\end{aligned}
$$

By the definition of $D_{-}^{\sigma}$, we see that, for fixed $x_{1} \in \Omega_{R_{\varepsilon}}$, there exists a sufficiently small $\sigma_{1}$ such that $x_{1} \in D_{-}^{\sigma_{1}}$. However, we know that, for any $\sigma<\sigma_{1}, D_{-}^{\sigma_{1}} \varsubsetneqq D_{-}^{\sigma}$. This finding implies that $x_{1} \in D_{-}^{\sigma}$. This fact, combined with (2.7) and (2.8), shows that

$$
u\left(x_{1}\right) \leq \psi\left(\tau_{1}\left(\int_{\left|x_{1}\right|}^{\infty} s^{1-\lambda_{1}} k_{1}(s) d s\right)^{1 / 2}-\sigma\right)+M
$$

and

$$
\psi\left(\tau_{2}\left(\int_{\left|x_{1}\right|}^{\infty} s^{1-\lambda_{1}} k_{1}(s) d s\right)^{1 / 2}+\sigma\right) \leq u\left(x_{1}\right)+M
$$

Since (2.15)-(2.16) hold for any $\sigma<\sigma_{1}$, using the rank preservation of the limit of the function $\psi$, we obtain

$$
u\left(x_{1}\right) \leq \psi\left(\tau_{1}\left(\int_{\left|x_{1}\right|}^{\infty} s^{1-\lambda_{1}} k_{1}(s) d s\right)^{1 / 2}\right)+M
$$

and

$$
\psi\left(\tau_{2}\left(\int_{\left|x_{1}\right|}^{\infty} s^{1-\lambda_{1}} k_{1}(s) d s\right)^{1 / 2}\right) \leq u\left(x_{1}\right)+M
$$

These findings imply that, for any $x \in \Omega_{R_{\varepsilon}}$, the conditions in (2.14) hold.

Consequently, by Lemma B.2(ii), we have

$$
\begin{aligned}
& \limsup _{|x| \rightarrow \infty} \frac{u(x)}{\psi\left(\tau_{1}\left(\int_{|x|}^{\infty} s^{1-\lambda_{1}} k_{1}(s) d s\right)^{1 / 2}\right)} \leq 1, \\
& \liminf _{|x| \rightarrow \infty} \frac{u(x)}{\psi\left(\tau_{2}\left(\int_{|x|}^{\infty} s^{1-\lambda_{1}} k_{1}(s) d s\right)^{1 / 2}\right)} \geq 1 .
\end{aligned}
$$

For $C_{f} \in[1, \infty)$, it follows by Lemma B.2(iv) that

$$
\limsup _{|x| \rightarrow \infty} \frac{u(x)}{\psi\left(\left(\int_{|x|}^{\infty} s^{1-\lambda_{1}} k_{1}(s) d s\right)^{1 / 2}\right)}
$$




$$
\begin{aligned}
& \quad=\limsup _{|x| \rightarrow \infty} \frac{u(x)}{\psi\left(\tau_{1}\left(\int_{|x|}^{\infty} s^{1-\lambda_{1}} k_{1}(s) d s\right)^{1 / 2}\right)} \cdot \lim _{|x| \rightarrow \infty} \frac{\psi\left(\tau_{1}\left(\int_{|x|}^{\infty} s^{1-\lambda_{1}} k_{1}(s) d s\right)^{1 / 2}\right)}{\psi\left(\left(\int_{|x|}^{\infty} s^{1-\lambda_{1}} k_{1}(s) d s\right)^{1 / 2}\right)} \\
& \leq \tau_{1}^{1-C_{f}} ; \\
& \liminf _{|x| \rightarrow \infty} \frac{u(x)}{\psi\left(\left(\int_{|x|}^{\infty} s^{1-\lambda_{1}} k_{1}(s) d s\right)^{1 / 2}\right)} \\
& \quad=\liminf _{|x| \rightarrow \infty} \frac{u(x)}{\psi\left(\tau_{2}\left(\int_{|x|}^{\infty} s^{1-\lambda_{1}} k_{1}(s) d s\right)^{1 / 2}\right)} \cdot \lim _{|x| \rightarrow \infty} \frac{\psi\left(\tau_{2}\left(\int_{|x|}^{\infty} s^{1-\lambda_{1}} k_{1}(s) d s\right)^{1 / 2}\right)}{\psi\left(\left(\int_{|x|}^{\infty} s^{1-\lambda_{1}} k_{1}(s) d s\right)^{1 / 2}\right)} \\
& \geq \tau_{2}^{1-C_{f}} .
\end{aligned}
$$

Letting $\varepsilon \rightarrow 0$, we obtain (1.4).

\section{Proof of Theorem 1.2}

In this section, we prove Theorem 1.2.

Proof Since

$$
\lim _{|x| \rightarrow \infty} \frac{u_{1}(x)}{u_{2}(x)}=1
$$

for arbitrary fixed $\varepsilon>0$, there exists a sufficiently large $R_{\varepsilon}$ such that

$$
(1-\varepsilon) u_{2}(x) \leq u_{1}(x) \leq(1+\varepsilon) u_{2}(x), \quad x \in \Omega_{R_{\varepsilon}} .
$$

Set

$$
u^{+}(x)=(1+\varepsilon) u_{2}(x), \quad u^{-}(x)=(1-\varepsilon) u_{2}(x), \quad x \in \mathbb{R}^{N} .
$$

A simple calculation shows that

$$
\begin{aligned}
& \Delta u^{+}=(1+\varepsilon) \Delta u_{2}=(1+\varepsilon) a(x) f\left(u_{2}\right)+\mu(1+\varepsilon) b(x)\left|\nabla u_{2}\right|^{q}, \\
& \Delta u^{-}=(1-\varepsilon) \Delta u_{2}=(1-\varepsilon) a(x) f\left(u_{2}\right)+\mu(1-\varepsilon) b(x)\left|\nabla u_{2}\right|^{q} .
\end{aligned}
$$

By $\left(\mathbf{f}_{3}\right)$, we have

$$
(1+\varepsilon) f\left(u_{2}\right) \leq f\left((1+\varepsilon) u_{2}\right), \quad(1-\varepsilon) f\left(u_{2}\right) \geq f\left((1-\varepsilon) u_{2}\right) .
$$

In contrast, we apply $\left(\mathbf{b}_{1}\right)$ and $\left(\mathbf{b}_{3}\right)$ and conclude that

$$
(1+\varepsilon) b(x)\left|\nabla u_{2}\right|^{q} \leq b(x)\left|(1+\varepsilon) \nabla u_{2}\right|^{q}, \quad(1+\varepsilon) b(x)\left|\nabla u_{2}\right|^{q} \leq b(x)\left|(1+\varepsilon) \nabla u_{2}\right|^{q} .
$$

It follows by combining these with $\left(\mathbf{a}_{1}\right)$ that

$$
\Delta u^{+} \leq a(x) f\left(u^{+}\right)+\mu b(x)\left|u^{+}\right|^{q} ; \quad \Delta u^{-} \geq a(x) f\left(u^{-}\right)+\mu b(x)\left|u^{-}\right|^{q}, \quad x \in \mathbb{R}^{N} .
$$


Let $u_{0}$ be the unique solution of

$$
\Delta u=a(x) f(u)+\mu b(x)|\nabla u|^{q}, \quad x \in \Omega_{0},\left.u\right|_{\partial \Omega_{0}}=u_{1},
$$

where $\Omega_{0}=\mathbb{R}^{N} \backslash \Omega_{R_{\varepsilon}}$. Using the comparison principle, we obtain

$$
u^{-}(x) \leq u_{0}(x) \leq u^{+}(x), \quad x \in \Omega_{0} .
$$

Noting that $u_{0}=u_{1}$ on $\Omega_{0}$ and combining (3.1) with (3.2), we obtain

$$
(1-\varepsilon) u_{2}(x) \leq u_{1}(x) \leq(1+\varepsilon) u_{2}(x), \quad x \in \mathbb{R}^{N} .
$$

Letting $\varepsilon \rightarrow 0$ completes the proof.

\section{Appendix 1}

In this section, we introduce some preliminaries to Karamata regular variation theory.

Definition A.1 ([45], Definition 1.1) A positive continuous function $f$ defined on $[a, \infty)$ for some $a>0$ is called regularly varying at infinity with index $\rho$, written as $f \in R V_{\rho}$, if for each $\xi>0$ and some $\rho \in \mathbb{R}$,

$$
\lim _{s \rightarrow \infty} \frac{f(\xi s)}{f(s)}=\xi^{\rho} .
$$

In particular, when $\rho=0, f$ is called slowly varying at infinity.

Clearly, if $f \in R V_{\rho}$, then $L(s):=f(s) / s^{\rho}$ is slowly varying at infinity.

Some basic examples of functions that are slowly varying at infinity are

$\left(\mathrm{i}_{1}\right)$ every measurable function on $[a, \infty)$ with a positive limit at infinity;

$\left(\mathrm{i}_{2}\right)(\ln s)^{\beta}$ and $(\ln (\ln s))^{\beta}, \beta \in \mathbb{R}$; and

(i $\left.\mathrm{i}_{3}\right) e^{(\ln s)^{p}}, 0<p<1$.

Definition A.2 ([52], Definition 2.1) A positive continuous function $f$ defined on $[a, \infty)$ for some $a>0$ is called rapidly varying to infinity at infinity if for each $\rho>0$

$$
\lim _{s \rightarrow \infty} \frac{f(s)}{s^{\rho}}=\infty
$$

Some basic examples of functions that are rapidly varying to infinity at infinity are

(ii 1 ) $e^{s}$ and $e^{e^{s}}$;

(ii 2$) e^{e^{(\ln s)^{p}}}, e^{s^{p}}$ and $e^{e^{s^{p}}}, p>0$;

(ii 3$) s^{p} e^{(\ln s)^{p}}$ and $(\ln s)^{\beta} e^{(\ln s)^{p}}, p>1, \beta \in \mathbb{R}$; and

(ii 4$)(\ln s)^{\beta} e^{s^{p}}$ and $s^{\beta} e^{s^{p}}, p>0, \beta \in \mathbb{R}$.

Definition A.3 ([54], Definition 2.2) A positive continuous function $f$ defined on $(0, a]$ for some $a>0$ is called rapidly varying to infinity at zero if for each $\rho>0$

$$
\lim _{s \rightarrow 0^{+}} h(s) s^{\rho}=\infty
$$


Obviously, if we replace the variable $s$ in functions $\left(\mathrm{ii}_{1}\right)-\left(\mathrm{ii}_{4}\right)$ with the variable $s^{-1}$, we obtain basic examples of functions that are rapidly varying to infinity at zero.

In addition, it is not difficult to understand that a positive continuous function $g$ defined on $(0, a)$ for some $a>0$ is regularly varying at zero with index $\rho$ (written as $g \in R V Z_{\rho}$ ) if $s \rightarrow g(1 / s) \in R V_{-\rho}$. Similarly, $g$ is called rapidly varying at zero if $s \rightarrow g(1 / s)$ is rapidly varying at infinity.

Proposition 1 (Uniform convergence theorem; [41], Proposition 0.5) If $f \in R V_{\rho}$, then (A.1) holds uniformly for $\xi \in\left[c_{1}, c_{2}\right]$ with $0<c_{1}<c_{2}$.

Proposition 2 (Representation theorem; [45], Theorem 1.2; [41], Corollary, p. 17) A function $L$ is slowly varying at infinity if and only if it can be written in the form

$$
L(s)=\varphi(s) \exp \left(\int_{a_{1}}^{s} \frac{y(\tau)}{\tau} d \tau\right), \quad s \geq a_{1},
$$

for some $a_{1} \geq a$, where the functions $\varphi$ and $y$ are continuous and $s \rightarrow \infty, y(s) \rightarrow 0$ and $\varphi(s) \rightarrow c_{0}$ with $c_{0}>0$. If $\varphi \equiv c_{0}$, then $L$ is said to be normalized slowly varying at infinity and

$$
f(s)=s^{\rho} \hat{L}(s), \quad s \geq a_{1}
$$

is said to be normalized regularly varying at infinity with index $\rho$ (written as $f \in N R V_{\rho}$ ).

A function $f \in C^{1}\left[a_{1}, \infty\right)$ for some $a_{1}>0$ belongs to $N R V_{\rho}$ if and only if

$$
\lim _{s \rightarrow \infty} \frac{s f^{\prime}(s)}{f(s)}=\rho .
$$

Proposition 3 (Asymptotic behavior; [6], Propositions 1.5.8 and 1.5.10) If a function L is slowly varying at infinity, then, for $s \rightarrow \infty$,

$$
\int_{s}^{\infty} \tau^{\rho} L(\tau) d \tau \sim(-\rho-1)^{-1} \tau^{1+\rho} L(\tau), \quad \text { for } \rho<-1
$$

Proposition 4 ([6], Proposition 1.3.6) If a function $L$ is slowly varying at zero, then, for $s \rightarrow+\infty$,

$$
s^{\rho} L(s) \rightarrow+\infty, \quad s^{-\rho} L(s) \rightarrow 0, \quad \text { for } \rho>0 .
$$

\section{Appendix 2}

In this section, we collect some useful results.

Lemma B.1 ([53], Lemma 2.2) Letf satisfy $\left(\mathbf{f}_{\mathbf{1}}\right)$.

(i) Iff satisfies $\left(\mathbf{f}_{2}\right)$, then $C_{f} \geq 1$.

(ii) ( $\left.\mathbf{f}_{2}\right)$ holds for $C_{f} \in(1,+\infty)$ if and only iff $\in R V \frac{C_{f+1}}{C_{f^{-1}}}$.

(iii) If $\left(\mathbf{f}_{2}\right)$ holds for $C_{f}=1$, then $f$ is rapidly varying to infinity at infinity. 
Lemma B.2 ([53], Lemma 2.3) Letf satisfy $\left(\mathbf{f}_{\mathbf{1}}\right)-\left(\mathbf{f}_{\mathbf{2}}\right)$ and $\psi$ be the solution of problem (1.5).

Then

(i) $\psi^{\prime}(t)=-(2 F(\psi(t)))^{1 / 2}, \psi^{\prime \prime}(t)=f(\psi(t)), \psi(t)>0, t>0$;

(ii) $\lim _{t \rightarrow 0^{+}} \psi(t)=\infty$;

(iii) $\lim _{t \rightarrow 0^{+}} \frac{t \psi^{\prime \prime}(t)}{\psi^{\prime}(t)}=-C_{f}$; and

(iv) $\lim _{t \rightarrow 0^{+}} \frac{t \psi^{\prime}(t)}{\psi(t)}=1-C_{f}$, where $C_{f} \in[1, \infty)$.

Lemma B.3 (Comparison principle; [23], Theorems 10.1, 10.2) Let $\Psi(x, s, \xi)$ satisfy the following two conditions:

(D) $\Psi$ is nonincreasing in s for all $(x, \xi) \in \Omega \times \mathbb{R}^{N}$; and

( $\left.\mathbf{D}_{2}\right) \Psi$ is continuously differentiable with respect to the variable $\xi$ in $\Omega \times(0, \infty) \times \mathbb{R}^{N}$. If $u, v \in C(\bar{\Omega}) \cap C^{2}(\Omega)$ satisfies $\Delta u+\Psi(x, u, \nabla u) \geq \Delta v+\Psi(x, v, \nabla v)$ in $\Omega$ and $u \leq v$ on $\partial \Omega$, then $u \leq v$ in $\Omega$.

\section{Acknowledgements}

The authors are greatly indebted to the anonymous referees for the invaluable suggestions and comments which quite highly improved the quality of the presentation.

\section{Funding}

This work is partially supported by the RP of Shandong Higher Education Institutions (J17KA173), the NSF of P.R. China (11571295) and the NSF of Shandong Province (ZR2016AL03).

\section{Availability of data and materials}

Data sharing is not applicable to this article as no data were generated or analyzed during the current study.

\section{Competing interests}

The authors declare to have no competing interests.

\section{Authors' contributions}

The authors contributed equally in this article. They have all read and approved the final manuscript.

\section{Author details}

${ }^{1}$ School of Mathematics and Information Science, Yantai University, Yantai, P.R. China. ${ }^{2}$ School of Mathematics and Information Science, Shandong Institute of Business and Technology, Yantai, P.R. China.

\section{Publisher's Note}

Springer Nature remains neutral with regard to jurisdictional claims in published maps and institutional affiliations.

Received: 26 March 2018 Accepted: 27 November 2018 Published online: 03 December 2018

\section{References}

1. Alsaedi, R., Maagli, H., Zeddini, N.: Existence and global behavior of positive solution for semilinear problems with boundary blow-up. J. Math. Anal. Appl. 425, 818-826 (2015)

2. Alves, C.O., Santos, C.A., Zhou, J.: Existence and non-existence of blow-up solutions for a non-autonomous problem with indefinite and gradient terms. Z. Angew. Math. Phys. 66, 891-918 (2015)

3. Bandle, C., Giarrusso, E.: Boudnary blow-up for semilinear elliptic equations with nonlinear gradient term. Adv. Differ. Equ. 1, 133-150 (1996)

4. Bandle, C., Marcus, M.: 'Large' solutions of semilinear elliptic equations: existence, uniqueness and asymptotic behaviour. J. Anal. Math. 58, 9-24 (1992)

5. Bieberbach, L.: $\Delta u=e^{u}$ und die automorphen Funktionen. Math. Ann. 77, 173-212 (1916)

6. Bingham, N.H., Goldie, C.M., Teugels, J.L.: Regular Variation. Cambridge University Press, Cambridge (1987)

7. Cheng, K.S., Ni, W.M.: On the structure of the conformal scalar curvature equation on Rn . Indiana Univ. Math. J. 41, 261-278 (1992)

8. Cîrstea, F., Du, Y.: Uniqueness of the blow-up boundary solution of logistic equations with absorbtion large solutions of elliptic equations with a weakly superlinear nonlinearity. J. Anal. Math. 103, 261-277 (2007)

9. Cîrstea, F., Rădulescu, V.: Uniqueness of the blow-up boundary solution of logistic equations with absorbtion. C. R. Math. 335, 447-452 (2002)

10. Cîrstea, F., Rădulescu, V.: Blow-up boundary solutions of semilinear elliptic problems. Nonlinear Anal. 48, 521-534 (2002)

11. Cîrstea, F., Rădulescu, V:: Asymptotics for the blow-up boundary solution of the logistic equation with absorption. C. R. Acad. Sci. Paris, Ser. I 33, 231-236 (2003)

12. Cîrstea, F., Rădulescu, V.: Nonlinear problems with boundary blow-up: a Karamata regular variation theory approach Asymptot. Anal. 46, 275-298 (2006) 
13. Cîrstea, F., Rădulescu, V.: Boundary blow-up in nonlinear elliptic equations of Bieberbach-Rademacher type. Trans Am. Math. Soc. 359, 3275-3286 (2007)

14. Du, Y., Guo, Z:: Uniqueness and layer analysis for boundary blow-up solutions. J. Math. Pures Appl. 83, 739-763 (2004)

15. Du, Y., Huang, Q.: Blow-up solutions for a class of semilinear elliptic and parabolic equations. SIAM J. Math. Anal. 31 , 1-18 (1999)

16. Dupaigne, L., Ghergu, M., Goubet, O., Warnault, G.: Entire large solutions for semilinear elliptic equations. J. Differ. Equ. 253, 2224-2251 (2012)

17. García-Melián, J.: Boundary behavior for large solutions to elliptic equations with singular weights. Nonlinear Anal. 67, 818-826 (2007)

18. García-Melián, J., Rossi, J.D., Sabina de Lis, J.: A convex-concave elliptic problem with a parameter on the boundary condition. Discrete Contin. Dyn. Syst. 32, 1095-1124 (2012)

19. Ghergu, M., Rădulescu, V.: Existence and nonexistence of entire solutions to the logistic differential equation. Abstr. Appl. Anal. 17, 995-1003 (2003)

20. Ghergu, M., Rădulescu, V.: Nonradial blow-up solutions of sublinear elliptic equations with gradient term. Commun. Pure Appl. Anal. 3, 465-474 (2004)

21. Giarrusso, E.: Asymptotic behavior of large solutions of an elliptic quasilinear equation in a borderline case. C. R. Acad. Sci., Ser. 1 Math. 331, 777-782 (2000)

22. Giarrusso, E:: On blow up solutions of a quasilinear elliptic equation. Math. Nachr. 213, 89-104 (2000)

23. Gilbarg, D., Trudinger, N.S.: Elliptic Partial Differential Equations of Second Order, 3rd edn. Springer, Berlin (1998)

24. Guo, Z., Shang, J.: Remarks on uniqueness of boundary blow-up solutions. Nonlinear Anal. 66, 484-497 (2007)

25. Huang, S., Li, W., Tian, Q., Mu, C.: Large solution to nonlinear elliptic equation with nonlinear gradient terms. J. Differ. Equ. 25, 3297-3328 (2011)

26. Keller, J.B.: On solutions of $\Delta u=f(u)$. Commun. Pure Appl. Math. 10, 503-510 (1957)

27. Lair, A.V.: A necessary and sufficient condition for existence of large solutions to semilinear elliptic equations. J. Math. Anal. Appl. 240, 205-218 (1999)

28. Lair, A.V.: Nonradial large solutions of sublinear elliptic equations. Appl. Anal. 82, 431-437 (2003)

29. Lair, A.V., Wood, A.W.: Large solutions of semilinear elliptic equations with nonlinear gradient terms. Int. J. Math. Math. Sci. 22, 869-883 (1999)

30. Lazer, A.C., McKenna, P.J.: On a problem of Bieberbach and Rademacher. Nonlinear Anal. 21, $327-335$ (1993)

31. Lazer, A.C., McKenna, P.J.: Asymptotic behavior of solutions of boundary blowup problems. Differ. Integral Equ. 7, $1001-1019$ (1994)

32. Loewner, C., Nirenberg, L.: Partial differential equations invariant under conformal or projective transformations. In: Contributions to Analysis (a Collection of Paper Dedicated to Lipman Bers), pp. 245-272. Academic Press, New York (1974)

33. López-Gómez, J.: Optimal uniqueness theorems and exact blow-up rates of large solutions. J. Differ. Equ. 224, 385-439 (2006)

34. Mitidieri, E., Pohozaev, S.: A priori estimates and the absence of solutions of nonlinear partial differential equations and inequalities. Proc. Steklov Inst. Math. 234, 1-362 (2001)

35. Mohammed, A.: Existence and asymptotic behavior of blow-up solutions to weighted quasilinear equations. J. Math. Anal. Appl. 298, 621-637 (2004)

36. Mohammed, A.: Boundary asymptotic and uniqueness of solutions to the $p$-Laplacian with infinite boundary values. J. Math. Anal. Appl. 325, 480-489 (2007)

37. Ni, W.-M.: On the elliptic equation $\Delta u+K(x) u^{(n+2) /(n-2)}=0$, it's generalizations, and applications in geometry. Indiana Univ. Math. J. 31, 493-529 (1982)

38. Oleinik, O.A.: On the equation $\Delta u+k(x) e^{2 u}=0$. Russ. Math. Surv. 33, 243-244 (1978)

39. Osserman, R.: On the inequality $\Delta u \geq f(u)$. Pac. J. Math. 7, 1641-1647 (1957)

40. Rademacher, H.: Einige besondere problem partieller Differentialgleichungen. In: Die Differential und Integralgleichungen, der Mechanik und Physik I, pp. 838-845. Rosenberg, New York (1943)

41. Resnick, S.I.: Extreme Values, Regular Variation, and Point Processes. Springer, New York (1987)

42. Rhouma, N.B., Drissi, A.: Large and entire large solutions for a class of nonlinear problems. Appl. Math. Comput. 232, 272-284 (2014)

43. Santos, C.A., Zhou, J., Santos, J.A.: Necessary and sufficient conditions for existence of blow-up solutions for elliptic problems in Orlicz-Sobolev spaces (2016). arXiv:1601.01267

44. Sattinger, D.H.: Conformal metrics in $\mathbb{R}^{2}$ with prescribed curvature. Indiana Univ. Math. J. 22, 1-4 (1972)

45. Seneta, E.: Regular Varying Functions. Lecture Notes in Math, vol. 508. Springer, Berlin (1976)

46. Tao, S., Zhang, Z.: On the existence of explosive solutions for semilinear elliptic problems. Nonlinear Anal. 48, 1043-1050 (2002)

47. Wan, H.: Asymptotic behavior of entire large solutions to semilinear elliptic equations. J. Math. Anal. Appl. 448, 44-59 (2017)

48. Wan, H.: Asymptotic behavior and uniqueness of entire large solutions to a quasilinear elliptic equation. Electron. J. Qual. Theory Differ. Equ. 2017, 30 (2017)

49. Wan, H., Li, X., Li, B., Shi, Y.: Entire large solutions to semilinear elliptic equations with rapidly or regularly varying nonlinearities. Nonlinear Anal., Real World Appl. 45, 506-530 (2019)

50. Ye, D., Zhou, F.: Existence and nonexistence of entire large solutions for some semilinear elliptic equations. J. Partial Differ. Equ. 21, 253-262 (2008)

51. Zhang, Z:: Boudary behavior of large solutions to semilinear elliptic equations with nonlinear gradient terms. Nonlinear Anal. 73, 3348-3363 (2010)

52. Zhang, Z.: Boundary behavior of large solutions to the Monge-Ampére equations with weights. J. Differ. Equ. 259, 2080-2100 (2015)

53. Zhang, Z.: Boudary behavior of large solutions for semilinear elliptic equations with weights. Asymptot. Anal. 96, 309-329 (2016)

54. Zhang, Z., Li, B.: The boundary behavior of the unique solution to a singular Dirichlet problem. J. Math. Anal. Appl. 391, 278-290 (2012) 